\title{
The Role of Big Data in Intelligent Combat Command
}

\author{
Yuanli Qin ${ }^{a}$, Xunli Zhang , Guiqing Gao and Kang Wang \\ Xi'an High-tech Research Institute, Xi'an Shaanxi ,710025, China. \\ a897528959@qq.com
}

\begin{abstract}
With the development and application of high-tech, such as big data and artificial intelligence, the form of operational command has undergone qualitative changes, and intelligent combat operations are gradually becoming possible. This paper proposes the essence of the intelligent operational command. Starting with the analysis of the basic characteristics of big data technology, it reveals that big data technology is the key factor of intelligent operational command, and explores ways of thinking about intelligent command command building based on big data. It has guiding and reference functions for intelligent combat command and the use of new combat forces.
\end{abstract}

Keywords: big data, artificial intelligence, intelligent combat command.

\section{Introduction}

Big data and information have become the core elements of the transformation of combat effectiveness. At present, with the rapid development of big data and artificial intelligence technologies and the expansion and deepening of the application of intelligent weaponry in the military field, combat command is undergoing disruptive changes, and intelligence has become an inevitable trend for future war development. Mass data will become intelligent. With the advantages of combat command, how to identify, classify, excavate, and integrate information on the battlefield's Shanghai scale using big data technology, and then to form an accurate battle command has increasingly become the key to winning a new type of war.

\section{The Essence of Intelligent Combat Command}

\subsection{The Main Features of Intelligent Combat Command}

Intelligent operational command is a breakthrough in the traditional human-oriented combat decision-making method under the conditions of making full use of high-tech data such as big data and artificial intelligence, intelligent judgment and decision making, and the use of intelligent command terminals, networks, and other information systems to bring battlefields to the battlefield. The personnel, equipment, materials, and other operational, command, and support entities are closely linked to form a large battlefield data and perform multiple intelligence interpretation and information fusion through big data high-performance computing and high-efficiency algorithms to evaluate the battlefield situation., Optimize the operational plan, determine the combat determination, and implement combat command actions, and ultimately strike the target quickly and efficiently.

The typical characteristics of intelligent combat command: First, based on big data. Big data contains a growing amount of complex data, involving the acquisition, management and application of these key technologies and solutions for the entire life cycle of heterogeneous data. It is mainly used to obtain valid data, perform data analysis, realize data display and process data in real time. The second is based on artificial intelligence. Artificial intelligence is a discipline that enables machines to perform tasks that require human command, such as making assumptions, performing hypothesis verification, learning, and reasoning. It can enhance the effectiveness of war prejudgment, speed up the rhythm of warfare, and achieve speed to achieve victory. It can realize the autonomous confrontation of intelligent weapons and equipment, and guide the change of combat mode. It can break the traditional rules and methods of war to make clustered operations (centralized deployment on hundreds of Thousands of intelligent weapons, attacking targets from multiple directions) became possible. The third is based on supercomputing. Supercomputing is based on advanced clustering 
technology. It is a new computing model that has been developed along with the Internet and is specifically designed for complex scientific computing. It has powerful computing capabilities and consists of several supercomputer groups. It is mainly used to concentrate computing resources and achieve resource sharing, making the battlefield data and transparent.

\subsection{Intelligent Combat Command is an Inevitable Historical Development}

Combat command is a military practice activity aimed at maximizing and exerting operational command capabilities. It is divided into cold weapon operational command, hot weapon operational command, mechanized operational command, and informationized operational command. At present, big data, new algorithms, supercomputing, and artificial intelligence are affecting different areas of the war with unprecedented breadth and depth, promoting a new round of military reform, the shape and appearance of war is quietly being changed, and combat command is gradually being developed by information. From the transformation of intelligence to intelligence, its command methods and command methods will undergo a disruptive change, and the command theory will also be continuously updated and updated so that it can play its leading and guiding role more effectively in command activities. Therefore, in line with the development of the "smart +" era, focusing on how to effectively respond to the impact of the intelligent era, it is necessary to make better use of hightech cutting-edge technologies to achieve victory in war.

\subsection{Development Advantages of Intelligent Combat Command}

Intelligent operations will soon become a new military revolution. In recent years, the United States has extensively conducted artificial intelligence-based technology development and project research in various fields of air combat, focusing on intelligence reconnaissance and command and control. The most shocking thing is that on March 22, the latest short video released by the United States, "Air Force 2030 - Call It Now," has shown the new form of air combat in the future, pointing the way for future intelligent operations. Big data technology processes and analyzes massive intelligence data, and artificial intelligence technology assists commanders in decision-making, so that the war command system can be transformed and transformed in all directions.

(1) Battlefield Situation Intelligence Cognition. Based on the reconnaissance, perception, and technical means widely distributed in the multidimensional battlefield space, based on the real-time sharing of the battlefield situation, the battlefield data is intelligently processed to form intelligent knowledge awareness activities on battlefield military activities.

(2) Intelligent integration of combat forces. According to the actual needs of combat, with the help of highly shared information and rapid circulation, various operational systems, operational elements, and operational units that are dynamically distributed and deployed in a vast multidimensional battlefield space are automatically selected in real time to form an organic combat force as a whole, thereby giving full play to the combat system. Overall performance.

(3) Command and decision intelligence assistance. The intelligent auxiliary decision system proposes multiple sets of operational plans or plans for the commander to determine, the intelligent combat unit receives the combat missions and target requirements sent by the accusation center synchronously, and designs the optimal maneuvering, reconnaissance, attack, and security plans to achieve the command decision. The advancement of power will effectively save battlefield resources and improve the speed and quality of decision-making.

(4) Intelligent release of combat effectiveness. Under the support of the joint combat system, the intelligent combat unit automatically reconnaissances and recognizes target information according to operational tasks and requirements, and autonomously conducts precise offensive and defensive actions according to the nature, position, size, and status of the target, so as to achieve sophisticated release of combat effectiveness. 


\section{Big Data is a Key Factor Affecting Intelligent Battle Command}

\subsection{The Basis for the Implementation of Operational Command}

Before the information age, people relied on sampling data and local data, as well as experience, theory, and hypotheses to explore the laws of the unknown. Therefore, the perception of the battlefield was superficial, superficial, and simple. The advent of the era of big data has made data a new type of weapon, laying the foundation for further exploration and study of the laws of the battlefield, and providing the possibility for the realization of intelligent operational command.

(1) Provide information resources. Big data has the characteristics of diverse data types, highspeed data processing, massive data size, and low data value density. The massive information contained in big data and the analysis of acquired information, the mining of hidden information behind the information brings information advantages to the operational command;

(2) Mining the relevance of data information. The key technologies and processing systems included in Big Data are effective in rapidly collecting, analyzing, and processing disorderly and disorderly real-time battlefield information. Through the analysis of the correlation between battlefield data, they are analyzed from seeming contradictory information. Sex, to find out the rules of combat command and battlefield evolution; Figure 1 Big Data Mining process flow .

(3) Derived information. Big data technology stimulates the emergence of information from the data of massive systems, derives more available information on the basis of massive data, and forms information on the battlefield, which in turn leads to information advantages in combat commanding such as situation determination, command decision-making, and coordination and control.

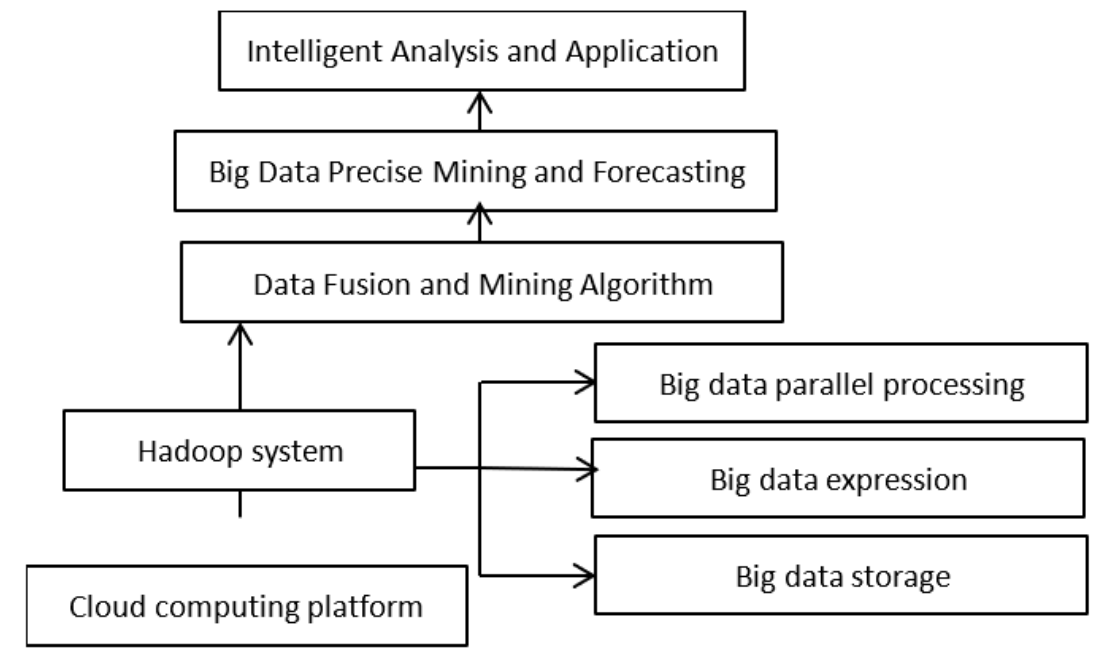

Figure 1.big data mining process

\subsection{Implementation of Accurate Command Guarantee}

The information collected by big data has huge volume and many types of information. High-value information is an important guarantee for effective command. Data information technology includes information collection, information transmission, information processing, and information display. The key technology is information analysis and processing technology. In the process of data processing, the corresponding methods are usually applied to the characteristics of data and the requirements of operational command and decision making. Initial processing of data, statistical analysis of the massive data collection and aggregation, so as to filter out valuable data information. Figure 2. Workflow diagram of the auxiliary command and decision system supported by big data.

Driven by the application of big data, combat commanders monitor and track the dynamics of the battlefield between the enemy and me through real-time monitoring, excavating and analyzing mass battlefield data, revealing the rules of combat command, and formulating more scientific and reasonable operational plans. In order to verify the correctness of the analysis results, big data analysis uses competition hypothesis analysis to establish the corresponding evaluation criteria, rules and 
knowledge base, and design the corresponding data mining algorithm, which provides a reliable guarantee for accurate command.

The information system under the support of big data technology can use the general situation map and other necessary intelligence information formats to access the massive battlefield information in accordance with the privilege and requirements. This enables all-source intelligence information acquisition and multi-level hierarchical information processing. From the bottom to the top, they are distributed vertically and distributed automatically from the top to the bottom. The real-time information sharing between the commanders at all levels and the end of the weapon ensures that the information is highly integrated with the firepower, hitting the target directly at the enemy's key points, and achieving accurate reconnaissance positioning and precise command. Control, accurate fire strikes and accurate performance evaluations to achieve precise implementation of command methods.

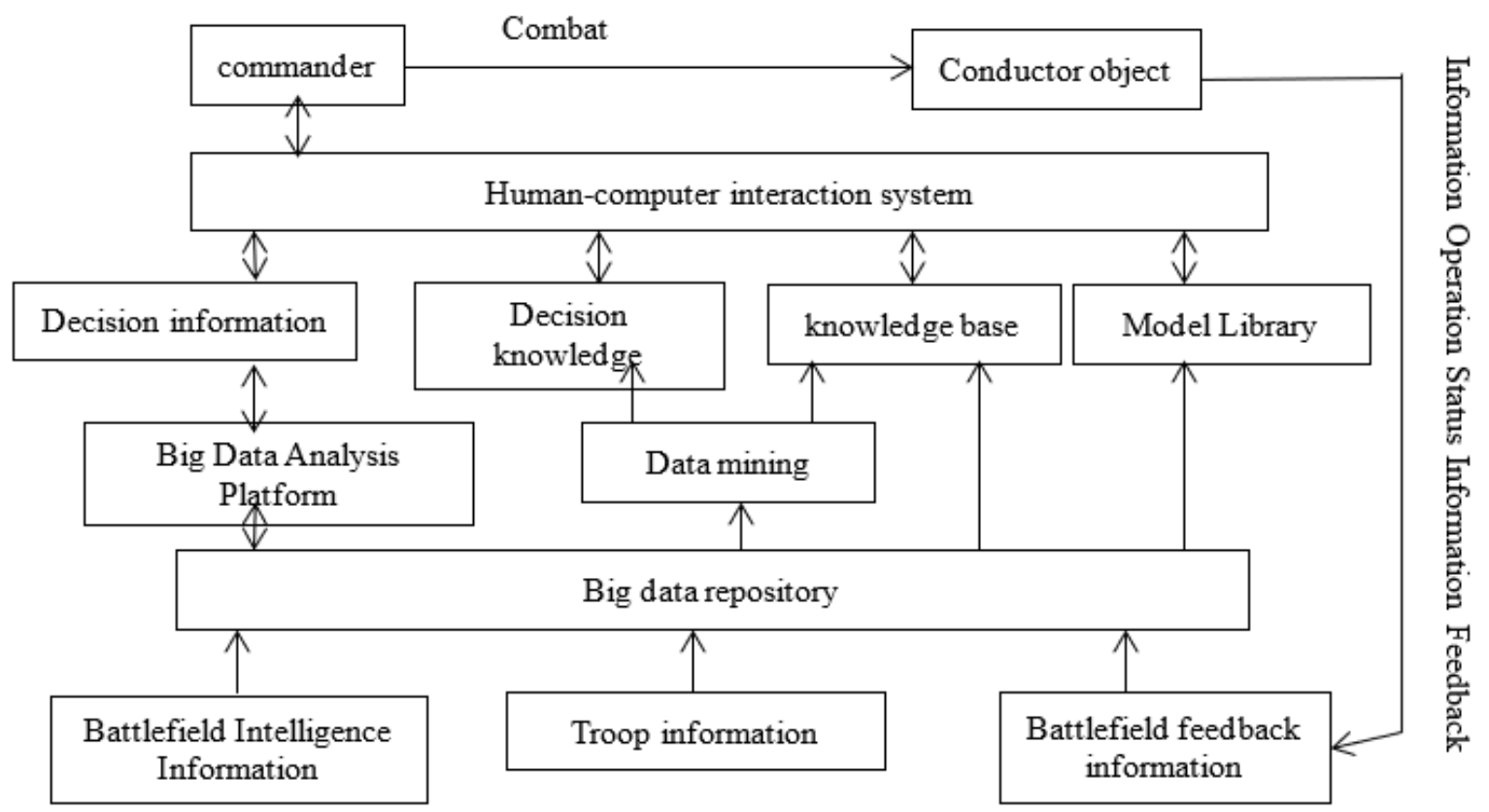

Figure 2.Workflow diagram of the auxiliary command and decision system supported by big data

\subsection{Basis for Establishing Command System}

Whether the advantages of information can be transformed into decision-making advantages to the maximum extent is a measure of whether the operational command and command system meets operational requirements. Big data technology uses the relationship between the integrated command platform and the command level and command span to analyze the relationship between command hierarchy and command span of the information architecture on the integrated command platform from the perspective of information flow, and studies the command hierarchy in different operational styles. Command relations so as to maximize the combat capabilities. The core of the U.S. cyberspace network center is to rely on the network to integrate disparate operational elements and form information advantages, and to maximize information advantages into decision-making and operational advantages.

Big data technology has also impacted the command method of the existing command system, making the operational command method "programmatic" to "non-procedural" highlights, enabling seamless linking of information transmission, information exchange and information sharing, and achieving a highly transparent battlefield. The coexistence of situations and self-synchronization of actions make it easier for commanders to make allegations of war operations. In the command method with centralized command as the main command, the middle command level can make full use of transparent information channels, combine their own authorities, and influence the command of the decentralized and optimize the concentrated command of the superior, so that the centralized 
command and the decentralized command show you there is me. , I have your situation in order to better adapt to the changing battlefield environment.

In the era of big data, technologies such as cloud computing, the Internet of Things, and artificial intelligence are fully used in the military field. Flat structure, simple hierarchy, high degree of integration, and system integration have become the development direction of the operational command system.

\section{Thinking about the Construction of Intelligent Combat Command Capability Based on Big Data}

\subsection{Establishing an Intelligent Decision System}

Combat command and decision-making is a series of planning, optimization, and decision-making actions conducted by the combat commander during the Minhang operations to determine the operational goals and methods of action of the troops. Traditional decision-making systems can only complete the analysis and use of structured data. In the context of big data, the decision-making system will support unstructured data at the same time, and the competition for "regarding data rights" will become increasingly fierce. In the future, various weapons and equipment platforms on the battlefield will be linked by data and the complex adaptive system will become the future. The mainstream of system confrontation.

The cross-recurring analysis capability of big data cross-replicates multi-channel information from different perspectives and different dimensions, helping commanders to more systematically and comprehensively analyze and make decisions more objectively and objectively. It can support and realize the comprehensiveness, accuracy, and validity of information. It can effectively break the "fog of war" in the decision-making process, promote data from "assisted decision making" to "support decision-making," and expands the collection channels of decision information, making The seemingly unrelated data acquisition is necessary, making all kinds of intelligent terminals a source of battlefield information. With efficient information fusion and effective information sharing, battlefield information can be efficiently operated and integrated. At present, major breakthroughs in some high and new technologies have made intelligent military decision-making a new possibility. For example, neural networks, expert systems, fuzzy logic, information fusion technologies, and so on.

The command and decision-making of intelligent operations is to give full play to the respective advantages of the human brain and machines under the support of reasonable people and division of labor and high-efficiency human-computer interaction, and to carry out situation analysis and judgment and set operations in a fast, accurate, efficient, and flexible manner. Determined to issue operational orders and conduct combat operations. The human brain can handle artistic and highlevel tasks such as high-level decision making and master planning. The machine processes massive, accurate, and high-speed data information memory, calculation, and management tasks. The effective integration between the two can complement each other and maximize the effectiveness of operational command decisions.

\subsection{Building a Battlefield Neural Network}

Neural network technology is to imitate the function of information transfer between human neurons, establish artificial neural network structure, and use computers to simulate human brain thinking to analyze, process, process, and utilize input information. The so-called battlefield neurosensory network system is to compare the battlefield to the human nervous system and use the information collected by big data to link the battlefields. It contains numerous battlefield information network nodes, and all information flow processes are intelligent, combined with artificial intelligence technology. IntelliSense function of the entire combat command system. Therefore, it is possible to predict and analyze the battlefield situation in real time, intelligently screen intelligence 
information, rely on massive data analysis and processing, allocate battlefield resources more scientifically and reasonably, and effectively exert the effectiveness of command and decision making.

\subsection{Building a Disruptive Weapon System}

At present, because weapons and equipment are developed, produced, assigned, used, and protected by different units and personnel, equipment information is prone to accumulation, loss, and duplication. Each branch is building its own weapons and equipment database. Data includes weapons and equipment. The model, performance, number of quality conditions, use, security and so on. With the rapid development and deep application of cloud computing, big data, artificial intelligence and mobile computing, the storage, processing, analysis, and integration of massive data have become innovation breakthroughs and development potentials for equipment development. In the field of weapons and equipment, relying on computer data management systems for business processing, such as equipment support data collection, query and application, whether from the perspective of modern warfare requirements on comprehensive and rapid information control, or from the perspective of database technology development trends, It is necessary to establish a powerful disruptive system that integrates the data that exists in the distributed data sources and provides comprehensive data collection and data support for the integrated department. On the one hand, it can make full use of existing systems and reduce the duplication of labor and investment brought about by the reconstruction system. On the other hand, the structural features of multiple databases facilitate the expansion of systems and system upgrades, and are conducive to the subsequent development and development of equipment data systems. The analysis of aid decision-making in the management and use of weaponry has been brought to a new level.

\section{References}

[1]. CAO Huizhi, LI Pei, LIU Junjie, etc. Research on the Construction and Development of Equipment Support under the Background of Big Data Era[J] China Information Management 2014(17):52-54.

[2]. Xu Shiyong, Wang Jiasheng, Exploring the winning mechanism of intelligent combat [J] Liberation Army Daily 2018 (007).

[3]. Zhao Shi, Big Data and Its Impact on Information Warfare Command[J] Information Technology and Informatization 2016(4):92-93.

[4]. Han Peng, Xu Chuncong, Liu Yu, etc., Big data analysis technology and its application in the military field [J] Computer Knowledge and Technology 2018(14):7-8.

[5]. Liu Yingfang, The embodiment of big data and its technology in the field of combat command [J] Technology Innovation and Application 2017(16): 293.

[6]. Lei Yi, Wang Jinsong, Yang Mingxi, The use of big data in information operations command and command control and simulation 2016(3):25-27.

[7]. Shen Shoulin, Zhang Guoning, Understanding Intelligent Operations [J] Liberation Army Daily 2018 (007).

[8]. Chen Hanghui Artificial Intelligence: How to Subvert the Future War China Defence News 2018(004). 